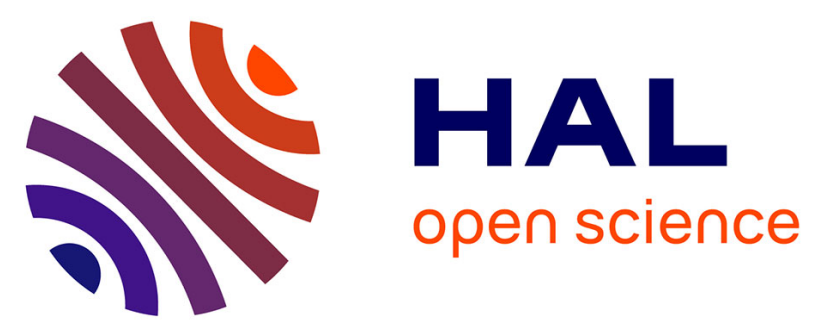

\title{
B-vitamin intake from diet and supplements and breast cancer risk in middle-aged women: Results from the prospective NutriNet-Santé cohort
}

Manon Egnell, Philippine Fassier, Lucie Lécuyer, Laurent Zelek, Marie-Paule Vasson, Serge Hercberg, Paule Latino Martel, Pilar Galan, Mélanie Deschasaux, Mathilde Touvier

\section{To cite this version:}

Manon Egnell, Philippine Fassier, Lucie Lécuyer, Laurent Zelek, Marie-Paule Vasson, et al.. Bvitamin intake from diet and supplements and breast cancer risk in middle-aged women: Results from the prospective NutriNet-Santé cohort. 40. Annual San Antonio Breast Cancer Symposium (SABCS 2017), Dec 2017, San Antonio, Texas, United States. American Association for Cancer Research, Cancer Research, 78 (4, Supplement), 2018, Cancer Research. 10.1158/1538-7445.SABCS17-P5-1202 . hal-01857982

\section{HAL Id: hal-01857982 https://hal.science/hal-01857982}

Submitted on 2 Jun 2020

HAL is a multi-disciplinary open access archive for the deposit and dissemination of scientific research documents, whether they are published or not. The documents may come from teaching and research institutions in France or abroad, or from public or private research centers.
L'archive ouverte pluridisciplinaire HAL, est destinée au dépôt et à la diffusion de documents scientifiques de niveau recherche, publiés ou non, émanant des établissements d'enseignement et de recherche français ou étrangers, des laboratoires publics ou privés. 


\section{San Antonio Breast Cancer Symposium}

Publication Number: P5-12-02

Title: B-vitamin intake from diet and supplements and breast cancer risk in middle-aged women: Results from the prospective NutriNet-Santé cohort

Manon Egnell ${ }^{1}$, Philippine Fassier ${ }^{1,2}$, Lucie Lécuyer ${ }^{1,2}$, Laurent Zelek ${ }^{1,2,3}$, Marie-Paule Vasson ${ }^{2,4}$, Serge Hercberg ${ }^{1,2,5}$, Paule Latino-Martel ${ }^{1,2}$, Pilar Galan ${ }^{1}$, Mélanie Deschasaux ${ }^{1,2}$ and Mathilde Touvier ${ }^{1,2}$. 'Sorbonne Paris Cité Epidemiology and Statistics Research Center (CRESS), U1153 Inserm, U1125, Inra, Cnam, Paris 13 University, Nutritional Epidemiology Research Team (EREN), Bobigny, France; ${ }^{2}$ French Network for Nutrition and Cancer Research (NACRe Network), Jouy-en-Josas, France; ${ }^{3}$ Avicenne Hospital, Assistance Publique-Hôpitaux de Paris, Bobigny, France; ${ }^{4}$ Clermont Université, Université d'Auvergne, UFR Pharmacie; Inra, UMR 1019, CRNH Auvergne; Centre Jean-Perrin, CHU Gabriel-Montpied, Unité de Nutrition, Clermont-Ferrand, France and ${ }^{5}$ Avicenne Hospital, Assistance Publique-Hôpitaux de Paris, Bobigny, France.

Body: Experimental studies suggest a protective effect of B-vitamins on breast cancer risk, potentially modulated by alcohol intake. However, epidemiological studies are limited, especially regarding non-folate B-vitamins. Furthermore, few of them included quantitative assessment of supplemental intake. This prospective study aimed at investigating the associations between intakes of B-vitamins (dietary, supplemental, total) and breast cancer risk. 27,853 women aged $\geq 45 \mathrm{y}$ from the NutriNet-Santé cohort (2009-2016) were included, with a median follow-up time of 4.2 years. Dietary data were collected using repeated $24 \mathrm{~h}$ records. A specific questionnaire assessed dietary supplement use over a 12-month period. A composition database of 8000 supplements was developed. Associations were characterized by multivariable Cox models. 462 incident breast cancers were diagnosed. Dietary $\left(\mathrm{HR}_{\mathrm{Q} 4 v s . \mathrm{Q} 1}=0.74(0.55,0.99)\right.$, P-trend=0.05), supplemental $\left(\mathrm{HR}_{\mathrm{Q} 4 v \mathrm{v} . \mathrm{Q} 1}=0.61(0.38,0.98), \mathrm{P}\right.$-trend=0.05) and total $\left(\mathrm{HR}_{\mathrm{Q} 4 \mathrm{vs} . \mathrm{Q} 1}=0.67(0.50,0.91)\right.$, P-trend=0.01) pyridoxine intakes were inversely associated with breast cancer risk. Total thiamin

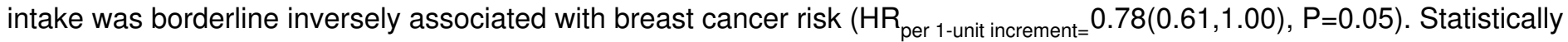
significant interactions between alcohol consumption and B-vitamin (thiamin, riboflavin, niacin, pantothenic acid, pyridoxine, folate, and cobalamin) supplemental intake were observed, the latter being inversely associated with breast cancer risk in non-to-low alcohol drinkers but not in higher drinkers. This large prospective study, including quantitative assessment of supplemental intake, suggests a potential protective effect of pyridoxine and thiamin on breast cancer risk in middle-aged women. 